\title{
TUMORES NEUROENDÓCRINOS DO CÓLON E RETO: EXPERIÊNCIA DO INSTITUTO NACIONAL DO CÂNCER NO BRASIL
}

\author{
Colon and rectum neuroendocrine tumors: experience of the National Cancer Institute in Brazil \\ Daniel CESAR, Renato Morato ZANATTO, Marcus Vinícius Motta Valadão da SILVA \\ Rinaldo GOLÇALVES, Eduardo Linhares Riello de MELLO, José Paulo de JESUS
}

Trabalho realizado no Departamento de Cirurgia Abdômino-Pélvica do Instituto Nacional do Câncer, Rio de Janeiro, RJ, Brasil.

DESCRTORES - Tumores neuroendócrinos. Cólon. Reto. Prognóstico. Tratamento.

\section{Correspondência:}

Daniel Cesar,

e-mail: danielcesar@live.com

Fonte de financiamento: não há Conflito de interesses: não há

Recebido para publicação: 20/09/2012 Aceito para publicação: 14/01/2013

HEADINGS - Neuroendocrine tumors Colon. Rectum. Prognosis. Treatment.
RESUMO - Racional - Os tumores neuroendócrinos (TNEs) são raros e compreendem apenas $0,49 \%$ de todas as neoplasias malignas. A maioria acomete o trato gastrointestinal. Objetivo - Analisar os fatores demográficos, características clinicopatológicas, tratamento empregado, fatores prognósticos e resultados oncológicos relacionados aos TNEs colorretais. Métodos - No período compreendido entre 1996 e 2010 foram tratados 174 pacientes. Destes, 34 localizavam-se no cólon e reto. Foram analisados fatores demográficos, estadiamento, os procedimentos terapêuticos aplicados e seus resultados. No seguimento todos os pacientes foram acompanhados acima de três anos com exames de imagem, dosagem de 5-HIIA urinário, cromogranina-A sérica e fosfatase ácida prostática. Resultados - A média de idade no diagnóstico foi de 54,5 anos (22-76), com predominância do sexo feminino (64,7\%). Dos 12 com TNEs de cólon, um (8,3\%) foi classificado como estádio IA; um (8,3\%) em IB; três (25\%) em IIB e sete $(58,4 \%)$ em IV. Dos 22 pacientes com TNEs de reto, seis $(27,3 \%)$ foram classificados como estádio IA; quatro (18,2\%) em IB; três (13,6\%) em IIIA; um (4,5\%) em IIIB e oito (36,4\%) em IV. Dos TNEs de reto, nove (41\%) foram tratados com ressecção endoscópica, seis $(27,2 \%)$ com procedimento cirúrgico e seis $(27,2 \%)$ somente com quimioterapia. Onze pacientes com TNEs de cólon (91,6\%), foram tratados cirurgicamente, sendo sete paliativamente, um (8,4\%) com ressecção endoscópica e nenhum foi submetido à quimioterapia no primeiro momento. Após seguimento médio de 55 meses, 19 (55\%) pacientes estavam vivos. Analisando a sobrevida global obteve-se média de 29 meses no estádio IA, 62 meses no IB, 12 meses no IIIA, 31 meses no IIIB e 39 meses no IV. Conclusão - O tratamento dos TNEs de cólon e reto é complexo, pois depende de variáveis que são individuais a cada paciente. Com adequado manuseio, o prognóstico pode ser favorável e a sobrevida longa, mas ela está relacionada ao grau de diferenciação tumoral, tamanho, localização do tumor, estadiamento no momento do diagnóstico, eficácia da terapêutica adotada e à aderência do paciente ao seguimento após o tratamento.

ABSTRACT - Background - Neuroendocrine tumors (NETs) are rare, comprising nearly $0.49 \%$ of all malignancies. The majority occurs in the gastrointestinal tract. $\mathbf{A i m}$ - To analyze the demographic factors, clinicopathologic features, treatment employed, prognostic factors and the oncologic results related to colorectal NETs. Methods - Between the period from 1996 to 2010174 patients were treated. From these, 34 were localized in the colon and rectum. Demographic factors, stage, therapeutics and its results were analyzed. All patients were followed for more than three years with image exams, urinary 5-hydroxyindolacetic acid (5-HIA), serum chromogranin A and prostatic acid phosphatase. Results - The median age was 54,4 years (22-76), the majority was female (64,7\%). Out of the 12 patients with colon NETs, one (8.3\%) patient was classified as Stage IA; one (8.3\%) as Stage IB; three (25\%) as Stage IIB and seven (58.4\%) as Stage IV. Out of the 22 patients with rectum NETs, six (27.3\%) were classified as Stage IA; four (18.2\%) as IB; three (13.6 $\%)$ as $\mathbb{I A}$; one (4.5\%) as IIB and eight (36.4\%) as IV. Of rectal NETs, nine (41\%) were treated with endoscopic resection, six (27.2\%) underwent conventional surgical treatment and six (27.2\%) were treated with chemotherapy. Eleven patients with colon NETs (91.6\%) were surgically treated, seven of them with palliative surgery, one (8.4\%) was treated with endoscopic resection and no patient was submitted to chemotherapy. After an average follow-up of 55 months, 19 (55\%) patients were alive. Analyzing the overall survival was obtained an average overall survival of 29 months in Stage IA, 62 months in IB, 12 months in IIA, 31 months in IIB and 39 months in IV. Conclusion - The treatment of colon and rectal NETs is complex, because it depends of the individuality of each patient. With adequate management, the prognosis can be favorable with long survival, but it is related to the tumor differentiation degree, efficacy of the chosen treatment and to the patient adhesion to the follow-up after treatment. 


\section{INTRODUÇÃO}

$\mathrm{O}$ s tumores neuroendócrinos são derivados das células de Kulchitsky ou cromafins que revestem o epitélio do trato respiratório e digestivo. São neoplasias raras 5 e compreendem apenas $0,49 \%$ de todos tumores malignos ${ }^{12}$. Tradicionalmente o termo "carcinóide" era empregado para designar os tumores epiteliais do intestino que apresentavam estrutura relativamente similar e comportamento menos agressivo que os carcinomas $^{14}$. Devido à heterogeneidade morfológica e biológica desta neoplasia, a Organização Mundial de Saúde (OMS) em 2000 adotou uma nova classificação passando a denominar essa neoplasia de Tumores Neuroendócrinos e Carcinomas Neuroendócrinos ${ }^{19}$. A distinção era feita entre tumores neuroendócrinos bem diferenciados que mostravam: 1) comportamento benigno ou potencial maligno incerto (classificação OMS 1a); 2) comportamento benigno com baixo grau de malignidade (classificação OMS 1b); 3) carcinomas neuroendócrinos bem diferenciados com baixo potencial maligno (classificação OMS 2); 4) carcinomas neuroendócrinos pouco diferenciados com alto potencial maligno (classificação OMS 3) ${ }^{9}$.

Apesar dos avanços consideráveis feitos nas últimas décadas em relação à origem celular, marcadores de proliferação celular, diagnóstico e tratamento dos TNEs do cólon e reto, alguns aspectos permanecem em aberto.

O objetivo do estudo foi analisar os fatores demográficos, características clinicopatológicas, tratamento empregado, fatores prognósticos e resultados oncológicos relacionados aos TNEs colorretais.

\section{MÉTODOS}

No período compreendido entre 1996 e 2010 foram tratados no Instituto Nacional do Câncer - INCA, Rio de Janeiro, RJ, Brasil, 174 pacientes com diagnóstico de tumor neuroendócrino do trato gastrointestinal. Destes, 34 localizavam-se no cólon e reto.

Para analisar os fatores demográficos foram observadas separadamente para os tumores do cólon e do reto a média de idade no diagnóstico e o gênero. Para o estadiamento utilizou-se a proposta da ENETS (European Neuroendocrine Tumor Society) de 200615. Quanto ao tratamento realizado foi estudado o emprego de procedimentos cirúrgicos endoscópicos, laparoscópicos, convencionais e o uso de terapia adjuvante.

No seguimento pós-tratamento, todos os pacientes foram acompanhados com consultas médicas aos três, seis, nove e 12 meses no primeiro ano, semestralmente durante os primeiros três anos e anualmente nos anos seguintes. Foram utilizados neste processo: USG ou TC abdome, radiografia de tórax, dosagem de 5-HIIA urinário, cromogranina-A sérica e fosfatase ácida prostática em todas as consultas.
Para prognóstico a recorrência foi definida como surgimento de doença regional ou à distância após três meses do tratamento inicial.

\section{RESULTADOS}

A média de idade no diagnóstico foi de 54,5 anos (22-76), com predominância do sexo feminino $(64,7 \%)$. Dos pacientes analisados, 12 (35,3\%) apresentaram TNEs de cólon e 22 (64,7\%) de reto. Quando analisada separadamente, a média de idade para TNEs de reto foi de 50 anos (22-74) com predomínio do sexo feminino $(72,7 \%)$, enquanto que para os do cólon foi de 59 anos (46-76) com distribuição idêntica entre os gêneros.

Os 34 pacientes foram estadiados pelo sistema proposto pela ENETS ${ }^{15}$. Dos 12 com TNEs de cólon, um $(8,3 \%)$ foi classificado como estádio IA; um $(8,3 \%)$ em IB; três (25\%) em IIIB e sete $(58,4 \%)$ em IV. Dos 22 pacientes com TNEs de reto, seis (27,3\%) foram classificados como estádio IA; quatro (18,2\%) em IB; três $(13,6 \%)$ em IIIA; um $(4,5 \%)$ em IIIB e oito $(36,4 \%)$ em IV.

Quando analisada a abordagem terapêutica dos TNEs de reto, nove (41\%) foram tratados com ressecção endoscópica, seis $(27,2 \%)$ com procedimento cirúrgico e seis $(27,2 \%)$ somente com quimioterapia por apresentarem doença à distância ou localmente avançada no momento do diagnóstico. Onze pacientes com TNEs de cólon (91,6\%), foram tratados cirurgicamente, sendo sete paliativamente uma vez que já se encontravam com doença avançada (estádio IV), um $(8,4 \%)$ com ressecção endoscópica e nenhum foi submetido à quimioterapia no primeiro momento.

Após seguimento médio de 55 meses (2-178 meses), 19 (55\%) pacientes com TNEs colorretais estavam vivos. Analisando a sobrevida global com relação ao estádio clínico, obteve-se sobrevida global média de 29 meses no estádio IA, 62 meses no IB, 12 meses no IIIA, 31 meses no IIIB e 39 meses no IV.

\section{DISCUSSÃO}

Em 2003, Modlin et al. ${ }^{12}$ analisaram 13.715 casos de TNEs coletados entre 1973 e 1999 onde a maior incidência de TNEs ocorreu no trato gastrointestinal (67.5\%), seguido pelos broncopulmonares (25.3\%). Quando analisados somente os TNEs do trato gastrointestinal, a maior parte ocorreu no intestino delgado (41.8\%), seguido por reto $(27,4 \%)$ e estômago $(8,7 \%)$. Aqui neste estudo foi encontrada a incidência de $19,5 \%$ de tumores de cólon e reto, portanto diferente dos porcentuais referidos por aqueles autores.

Média de idade relativamente mais baixa dos TNEs em relação aos adenocarcinomas já havia sido comprovada anteriormente por Godwin ${ }^{5}$, em 1975. O mesmo foi observado em pacientes desta série.

Os TNEs apresentam características morfológicas e padrão de coloração próprio. Suas células possuem 
citoplasma granular levemente rosado e núcleo arredondado, com poucas mitoses ${ }^{18}$. Foram batizadas de enterocromafins, porque são coradas por cromato de potássio. Absorvem e reduzem a prata, sendo também chamadas de argentafins. Algumas delas absorvem a prata, mas não são capazes de reduzi-la, recebendo a designação de argirofílicas².

Células argentafins e argirofílicas têm a habilidade de absorver e descarboxilar aminas precursoras. Tanto células da crista neural como os TNEs são capazes de sintetizar aminas muito similares e a habilidade dos TNEs em sintetizar 5-hidroxitriptofano (5-HTP) a partir do triptofano dos alimentos é patognomônico dessas neoplasias. O sub-produto do metabolismo do 5-HTP é o ácido 5-hidroxiindolacético (5-HIAA), um marcador usado na investigação clínica dos TNEs.

Inúmeras pesquisas vêm sendo feitas nos marcadores de proliferação celular, Ki-67, índice mitótico e proteína de supressão tumoral (p53) como preditores de comportamento maligno nos TNEs. A imunoistoquímica básica na avaliação e classificação histológica inclui Ki-67, cromogranina e sinaptofisina. Destes, a cromogranina foi utilizada neste estudo e mostrou-se adequada como sinalisadora de prognóstico.

Não há dúvida que os marcadores bioquímicos principais utilizados clinicamente nos TNEs são 5-HIIA urinário e a cromogranina-A sérica. $O$ grau de elevação do 5-HIIA urinário é correlacionado com a severidade da síndrome carcinóide. Mas, nos TNEs colorretais o exame é geralmente negativo ${ }^{4}$. A cromogranina-A sérica, embora não seja um marcador específico, é muito útil no seguimento, na avaliação de resposta e progressão de doença ${ }^{20}$. Concentrações séricas elevadas são encontradas em pacientes com doença avançada”, sendo, portanto, considerado marcador de prognóstico. Em TNEs do reto, o polipeptídeo pancreático, o enteroglucagon e $\beta$-hCG podem ser ferramentas úteis no seguimento dessa neoplasia ${ }^{13}$. A fosfatase ácida prostática está presente em $80-100 \%$ dos TNEs retais ${ }^{3}$; assim, esse marcador deve ser sempre solicitado uma vez feito o diagnóstico para fins de avaliação da resposta ao tratamento e seguimento pós-cirúrgico.

A apresentação clínica varia em função do tamanho, do sítio primário e do tipo de substância produzida pelo tumor. Existe associação entre doenças inflamatórias intestinais e TNEs colorretais ${ }^{6}$. Mas, na maioria das vezes, não estão associados a esta condição e geralmente são achados incidentalmente em exames endoscópicos, pois frequentemente são pequenos e assintomáticos. Quando se tornam maiores podem apresentar sangramento, obstrução intestinal e tenesmo. Embora variando em frequência, estes dados clínicos foram encontrados nos pacientes aqui tratados.

A síndrome carcinóide manifesta-se em aproximadamente $10 \%$ dos pacientes; ocorre quando os metabólitos do 5-HTP, neurohormônios e prostaglandinas liberados pelo tumor ganham a circulação sistêmica sem passar pelo metabolismo hepático. Ela ocorre na vigência de doença metastática hepática, doença retroperitoneal extensa ou quando o sítio primário é extra-intestinal (brônquio, ovários, testículos). É caracterizada por flush, diarréia, broncoespasmo, lesão de valvas cárdicas e pelagra1. Raramente TNEs de reto apresentam-se com síndrome carcinóide e, quando ocorre, os sintomas são semelhantes à síndrome carcinóide dos tumores de íleo devido a semelhança dos produtos secretados pelos dois tumores. Metástases à distância podem estar presentes no momento do diagnóstico $(1,7 \%-8,1 \%)$, mas na maioria dos casos a doença encontra-se localizada $\left(75 \%\right.$ - 85\%) ${ }^{12}$.

Grande partedos TNEs colorretais são diagnosticados por exame endoscópico. A ultrassonografia transrretal é método de imagem bastante útil no estadiamento para tumores retais. Pode estudar o tamanho do tumor, profundidade da invasão e linfonodopatias com boa acurácia ${ }^{11}$. Inclusive, propõe o tipo de tratamento baseado nos achados. Tumores pequenos $(<10 \mathrm{~mm})$, limitados à mucosa e sem doença nodal podem ser tratados por ressecção endoscópica ou excisão local com segurança14.

A tomografia computadorizada e ressonância magnética são mais sensíveis e específicas que a ultrassonografia para diagnóstico e seguimento em paciente com TNEs colorretais ${ }^{8}$. A cintilografia com octreotídeo (Octreoscan) é útil no diagnóstico de doença metastáticas não visualizadas nos exames anteriores ou para investigação de doença hepática onde o TNE primário é desconhecido.

Em novembro de 2006, o consenso da ENETS (European Neuroendocrine Tumor Society) aprovou a classificação TNM para o estadiamento dos TNEs do cólon e reto ${ }^{15}$. Esta deve ser a classificação a ser utilizada no manuseio desses pacientes para orientar a forma de tratamento e o prognóstico, a qual está relacionada ao grau de diferenciação tumoral, tamanho, localização do tumor, estadiamento no momento do diagnóstico, eficácia da terapêutica adotada e ao seguimento após tratamento.

O tratamento cirúrgico é o único capaz de curar TNEs de cólon e reto ${ }^{10}$. As opções cirúrgicas estão divididas em duas modalidades: operação convencional (colectomia, ressecção anterior do reto ou amputação abdominoperineal) e ressecção local (ressecção endoscópica, excisão local transanal ou TEM - Transanal Endoscopic Microsurgery). Em tumores menores que $10 \mathrm{~mm}$ a ressecção local é opção terapêutica aceitável, desde que o estudo com imagem não mostre linfoadenopatia, as margens obtidas sejam livres e grau de diferenciação não revele carcinoma neuroendócrino pouco diferenciado e/ou grau $3^{17}$. Em tumores maiores que $20 \mathrm{~mm}$, com invasão da muscular própria ou suspeita de doença linfonodal a operação convencional está indicada ${ }^{16}$. Os tumores entre $10-20$ mm são alvos de controvérsia. Nestes casos é fundamental diferenciarse o tratamento entre os TNEs de colon e reto. Em TNEs do cólon está indicada ressecção cirúrgica do segmento acometido, bem como de toda a cadeia linfonodal regional, quer por laparoscopia quer por laparotomia. Em TNEs do reto com invasão da camada muscular e 
angiolinfática ou que apresentem sintomas e que, por conseguinte, possuem pior prognóstico, procedimento cirúrgico aberto é melhor indicado. Os autores deste estudo sugerem que tumores maiores que $20 \mathrm{~mm}$ e com invasão linfovascular possuem maior risco de metástases, tanto linfonodal quanto à distância.

Na tentativa de preservação anal, diâmetro de 15 $\mathrm{mm}$ pode ser usado como ponto de corte para decisão entre excisão local ou amputação abdominoperineal, quando o tumor não apresentar invasão linfática ou vascular em biopsia prévia. Assim, o tratamento dos TNEs de reto maiores que $10 \mathrm{~mm}$ deve ser abordado individualmente, considerando-se idade, co-morbidade e desejo do paciente.

Nas Figuras 1 e 2 os autores sugerem algoritmos para o manuseio dos TNEs afim de melhor entender-se da abordagem terapêutica desses tumores.

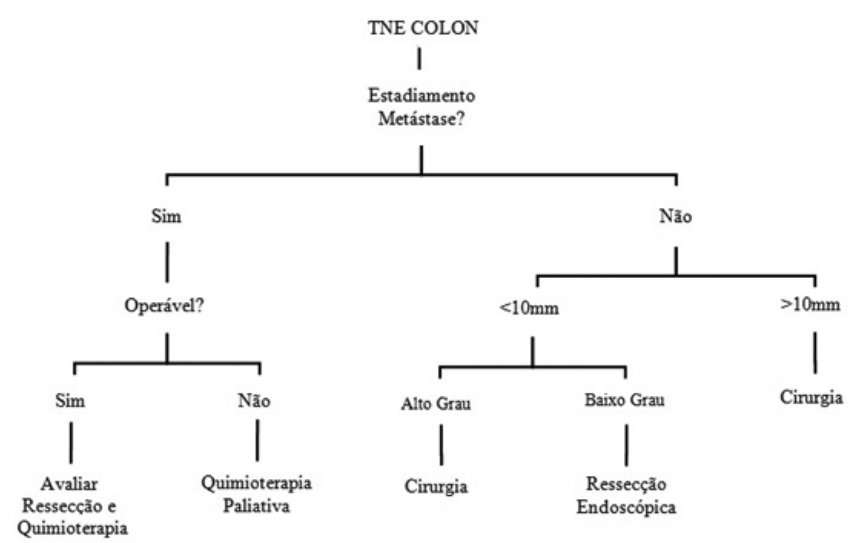

FIGURA 1 - Algoritmo para o tratamento TNEs de cólon

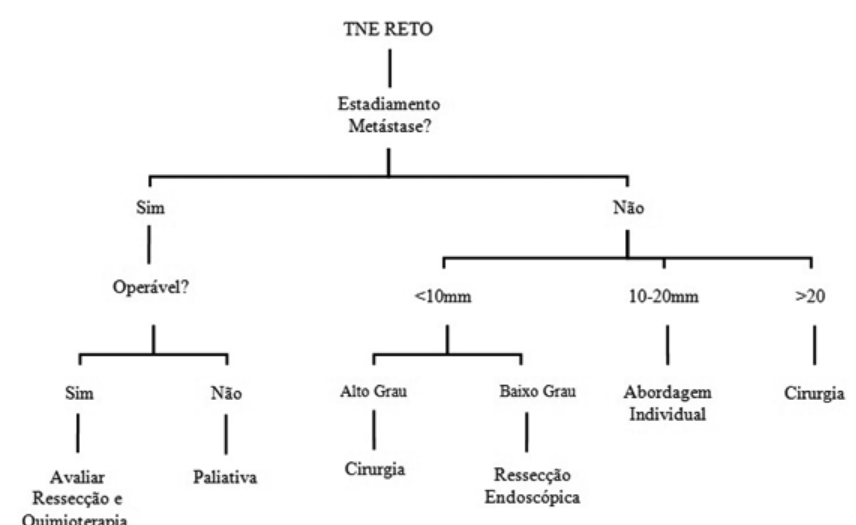

FIGURA 2 - Algoritmo do tratamento TNEs de reto

\section{CONCLUSÕES}

O tratamento dos TNEs de cólon e reto é complexo, pois depende de variáveis que são individuais a cada paciente. Com adequado manuseio o prognóstico pode ser favorável e a sobrevida longa, mas ela está relacionada ao grau de diferenciação tumoral, tamanho, localização do tumor, estadiamento no momento do diagnóstico, eficácia da terapêutica adotada e à aderência do paciente ao seguimento após o tratamento.

\section{REFERÊNCIAS}

1. Davis Z, Moertel CG, McIlrath DC. The malignant carcinoid syndrome. Surg Gynecol Obstet 1973; 137:637-44.

2. DeLellis RA, Dayal Y, Wolfe HJ. Carcinoid tumours: changing concepts and new perspectives. Am J Surg Pathol 1984; 8:295-300.

3. Federspiel $B H$, Burke AP, Sobin LH, Shekitka KM: Rectal and colonic carcinoids. A clinicopathologic study of 84 cases. Câncer 1990; 65: 35-140.

4. Feldman JM: Carcinoid tumors and the carcinoid syndrome. Curr Probl Surg 1989; 26:835-885.

5. Godwin JD 2nd. Carcinoid tumors. An analysis of 2,837 cases. Cancer 1975; 36:560.

6. Greenstein AJ, Balasubramanian S, Harpaz N, Rizwan M, Sachar DB: Carcinoid tumor and inflammatory bowel disease: a study of eleven cases and review of the literature. Am J Gastroenterol $1997 ; 92: 682-685$.

7. Janson ET, Holmberg L, Stridsberg $M$, et al.: Carcinoid tumors: Analysis of prognostic factors and survival in 301 patients from a referral center. Ann Oncol 1997; 8:685-690.

8. Jetmore $A B$, Ray JE, Gathright JB Jr, McMullen KM, Hicks TC, Timmcke AE: Rectal carcinoids: the most frequent carcinoid tumor. Dis Colon Rectum 1992; 35:717-725.

9. Klöppel G, Perren A, Heitz P. The Gastroenteropancreatic Neuroendocrine Cell System and Its Tumors: The WHO Classification. Ann. N.Y. Acad. Sci 2004; 1014:13-27.

10. Mani S, Modlin IM, Ballantyne G, Ahlman H, West B: Carcinoids of the rectum. J Am Coll Surg 1994; 179:231-248.

11. Matsumoto T, Iida M, Suekane $H$, Tominaga M, Yao T, Fujishima M: Endoscopic ultrasonography in rectal carcinoid tumors: contribution to selection of therapy. Gastrointest Endosc 1991; 37:539-542.

12. Modlin I, Lye K, Kidd M. A 5-decade analysis of 13,715 carcinoid tumors. Cancer 2003; 97:934-59.

13. Norheim I, Oberg $K$, Theodorsson-Norheim $E$, Lindgren PG, Lundqvist G, Magnusson A, Wide L, Wilander E: Malignant carcinóide tumors. An analysis of 103 patients with regard to tumor localization, hormone production, and survival. Ann Surg 1987; 206:115-125.

14. Oberndorfer S. Karzenoide tumoren des du"nndarms. Frankf Zschr Path. 1907; 1:426-430.

15. Rindi G, Klöppel G, Couvelard A, Komminoth $P, M$. Körner $M$ Lopes JM, McNicol AM, Nilsson O, Perren A, Scarpa A, Scoazec JY, Wiedenmann B. TNM staging of midgut and hindgut (neuro) endocrine tumors: a consensus proposal including a grading system. Virchows Arch 2007; 451:757-762.

16. Shebani KO, Souba WW, Finkelstein DM, Stark PC, Elgadi KM, Tanabe $\mathrm{KK}$, Ott MJ: Prognosis and survival in patients with gastrointestinal tract carcinoid tumors. Ann Surg 1999; 229:815-821.

17. Shields CJ, Tiret E, Winter DC. Carcinoid tumor of the rectum - a multi-institucional international collaboration. Ann Surg 2010; 252:750-755

18. Soga J, Tazawa K. Pathologic analysis of carcinoids. Cancer 1971; 28:990-98.

19. Solcia E, Klöppel G, Sobin LH. Histological typing of endocrine tumours. WHO International Histological Classification of Tumours. 2 ed. Berlin: Springer 2000.

20. Taupenot L, Harper KL, O'Connor DT. The chromograninsecretogranin family. N Engl J Med 2003; 348:1134-1149. 